\title{
Leaf Disease Detection
}

\section{Rithanayaa.K.A ${ }^{1}$, Atchaya.R ${ }^{2}$, Nandhitha.P.P ${ }^{3}$, Dr. S. Prakash ${ }^{4}$, Mr. B. Varun Kumar ${ }^{5}$, Ms. Gopika Venu ${ }^{6}$, Dr. R. P. S.Manikandan ${ }^{7}$}

1,2\&3 B.Tech Dept. of Information Technology, Sri Shakthi Institute of Engineering and Technology (Autonomous Institution), Coimbatore, Tamil Nadu, India.

${ }^{4}$ Professor, Sri Shakthi Institute of Engineering and Technology (Autonomous Institution), Coimbatore, Tamil Nadu, India.

5,6 Assistant Professor, Sri Shakthi Institute of Engineering and Technology (Autonomous Institution), Coimbatore, Tamil Nadu, India.

${ }^{7}$ Associate Professor, Sri Shakthi Institute of Engineering and Technology (Autonomous Institution), Coimbatore, Tamil Nadu, India.

$* * * * * *$

\begin{abstract}
:
Leaf disease detection is the process that help the users to detect the disease of the plant leafs. It is not only useful for farmers but also useful for plant researchers. Now a days agriculture wealth has been increased in each and every country particularly the agricultural strength in main in rural areas. But the technology hasnet inproved in rural area so people over there certainly not educated about plant disease. That causes heavy loss of plants and some of the disease can pass through one plant to another. That cause aheavy loss of plant as it gives a great loss for farmers. But we can prevent them by using this application.

The primary obejective of this project to identify and detect the leaf disease from leaf image data using image processing approach, which is implemented using python. To perform this task, this work used convolution Neural network $(\mathrm{CNN})$ algorithm for identify the leaf disease based on their unique physi3al shapes through geometric feather and venation architectures.Both real time and live detection of disease is done for the leafsamples.
\end{abstract}




\section{International Journal of Scientific Research in Engineering and Management (IJSREM)}

\section{INTRODUCTION}

The herbal plants in India is around 8,000 and $95 \%$ are collected from the forest area around India . To be generic $41 \%$ herbs including grasses, $26 \%$ trees, $17 \%$ shrubs, $16 \%$ climbers are seen across the country. But with the increasing population and extension of urban areas, the extinct situation is a threat for the plant generic resources. Disease affected the plants have a serious impact on Indian economy as they reduce the quantity and quality of the crops. Vegetables like tomato, potato ,cotton and capsicum are largely consumed in India and there is always an increasing demand for food supply. Therefore, it becomes at most important to protect the crops from various diseases that affect them thereby reducing the yield and as such we cannot afford to lose more of the food supply to crop diseases. These Diseases may be due to $\mathrm{pH}$ of soil, moisture content, temperature etc. The losses due to diseases can be catastrophic or chronic. Some of the disease include early blight, late blight and leaf mold. In olden days, identification is done manually or spectroscopically or microscopically by the experienced people but this is very time consuming and tedious job. Protecting the plants from disease is crucial, the diseases have high possibility of spreading and destroying other crops too. Hence, it is necessary forearly detection and identification of disease so as to help in choosing the correct treatment. Correct identification of disease is required so that apt measures like correct pesticide can be used for curing them. Detection of plant disease through some automatic technique is beneficial as it reduces a large work of monitoring in big farms of crops, and at very early stage itself it detects the symptoms of diseases i.e., when they appear on plant leaves. This paper presents an algorithm for image segmentation technique which is used for automatic detection and classification of plant leaf diseases. It also covers survey on different diseases classification techniques that can be used for plant leaf disease detection. Image segmentation, which is an important aspect for disease detection in plant leaf disease, is done by using Discrete \& Convolution algorithm. 


\section{LITERATURE REVIEW}

There are many different methods for leaf image classification. (Yin et al. 2014) adopted multispectral image techniques for categorizing green leaves. The idea was to use the entropy value of green tea leaf images as texture features. With full training, a support vector machine (SVM) with radial basis function (RBF) kernel successfully identifies the class labels than raw RBF.

This work has provided better segmentation rate in uncontrolled environments than the segmentation rate obtained by a color index technique by using a Baysian classifier using the two histogram models.

(Ahonen \& Pietikäinen 2007) (Mouine, Yahiaoui \& Verroust-Blondet 2013) proposed an original method for plant species recognition based on leaf observation. It uses TSL,SC2 and multi triangular approaches of a scanned image by a digital camera with untexturedbackround. Though the leaf color (Bai \& Ren 2011) is not sufficiently discriminant to be used alone in a plant identification task. Basically, this method uses SC2descriptor with a more accurate description of the leaf margin and leaf boundary.

Automated leaf image detection literature involves statistical feature matching approaches (An et al. 2016; Chen, Q et al. 2008; Kirk et al.2009) for appropriate edge detection. More semantic edge boundaries shall be identified using (An et al. 2016) whichis learned over very large datasets (Sun et al. 2017).

Recently, various deep learning-based plant leaf identification methods have been discussed in the literature. (Liu, H et al. 2014) applied a traditional CNN for feature extraction to apply over SVM for leaf classification. (Grinblat et al. 2016) (Hu et al. 2018) used vein pattern segmentation at the input of CNN for better classification accuracy. The chosen features of CNN were validated by a deconvolutional network in most literature.

\section{SYSTEM DEVELOPMENT:}

During which the design of a system is tested implementation is the stage of a project, then it is debugged and made it operational. Hence, this is the most crucial stage which in achieving a successful new system and the users in giving confidence that the system which is new will work and be more effective. 


\section{CLASSIFICATION USING CNN:}

In mostly neural networks, Convolutional neural network (ConvNets or CNNs) is one of the important categories to do recognition of images and classifications of images. CNN image classifications will take an input image, and process it and do classification under certain main categories. Convolution process is the first Layer to extract main features from an image at input. The relationship between pixels preserves by convolution by features using small squares of input data for learning image. It is a mathematical analysis operation that takes two basic inputs such as image in matrix and a filter or kernel form.

\section{PREDICTION:}

It $^{\text {ee }} \mathrm{s}$ a process of predicting the disease that what attack in a leaf. This project will effectively predict the data from dataset by enhancing the performance of the overall prediction results.

\section{PROPOSED SYSTEM:}

The primary objective of this project is to identify and detect the leaf disease from leaf image data using image processing approach, which is implemented using Python. To perform this task, this work used Convolutional Neural Network (CNN) algorithm for identify the leaf disease based on their unique physical shapes through geometric features and venation architectures. Both real-time and live detection of disease is done for the leaf samples. The proposed $\mathrm{CNN}$ algorithm isperformed better than existing techniques.

\section{HARDWARE COMPONENTS:}

- PROCESSOR : Intel Core i3.

- $\quad$ RAM :4GB

- MONITOR:15” COLOR

- HARD DISK:100GB 


\section{SOFTWARES USED:}

- Operating system: Windows 10

- Language: Python

- Front End IDE: Android Studio

- Back End: SQLITE

\section{FLOW CHART}
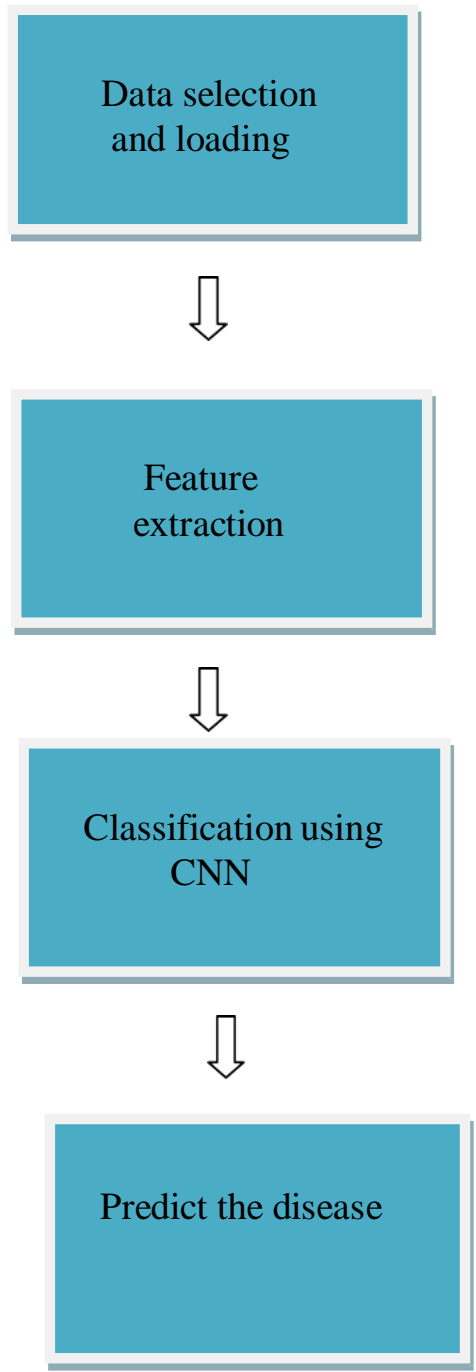


\section{CONCLUSION}

The current study developed Deep Learning for detecting leaf disease for farmers. The continuous video stream is read from the system and is used for detecting leaf disease.It is detected by using the convulusional neural network algorithm. The convulational neural network algorithm uses to detect classfication of image. CNN features are predefined are used for detecting different things. The CNN features are applied to the image or manager. Processing a deep learning yields additional computational complexities and hence certain other hybrid methodologies are necessitated in future for leaf disease detection.

\section{REFERENCE:}

1. Ben-Hur, D. Horn, H. T. Siegelmann, V. Vapnik, "Support vector clustering”, Journal of machine learning research, vol. 2, pp. 125-137, Dec 2011.

2. Gavhale, K.R., Gawande, U., ,An Overview of the Research on Plant Leaves Disease Detection using Image Processing Techniquesse, IOSR Journal of Computer Engineering, Volume 16, Issue 1, pp. 10-16, 2014

3. J. R. Otukei, T. Blaschke, "Land cover change assessment using decision trees support vector machines and maximum likelihood classification algorithms", International Journal of Applied Earth Observation and Geoinformation, vol. 12, pp. S27-S31, 2010.

4. J. R. Otukei, T. Blaschke, "Land cover change assessment using decision trees support vector machines and maximum likelihood classification algorithms", International Journal of Applied Earth Observation and Geoinformation, vol. 12, pp. S27S31, 2010

5. Jun Wu, Anastasiya Olesnikova, Chi-Hwa Song, Won Don Lee (2009). The Development and Application of Decision Tree for Agriculture Data. IITSI :16-20.

6. Mukhopadhyay S.C. Smart Sensing Technology for Agriculture and Environmental Monitoring. Vol. 146, Springer Berlin Heidelberg, (2012).

7. R.E. Schapire, "The boosting approach to machine learning: An overview" in Nonlinear estimation and 


\section{International Journal of Scientific Research in Engineering and Management (IJSREM)}

classification, New York: Springer, pp. 149-171, 2003.

8. Saradhambal, G., Dhivya, R., Latha, S., Rajesh, R., „Plant Disease Detection and its Solution using Image Classification $^{\text {ee }}$ International Journal of Pure and Applied Mathematics, Volume 119, Issue 14, pp. 879-884, 2018

9. Singh, J., Kaur, H., „A Review on: Various Techniques of Plant Leaf Disease Detection ${ }^{\text {ee }}$, Proceedings of the Second International Conference on Inventive Systems and Control, Volume 6, pp. 232-238, 2018.

10. Suma V, R Amog Shetty, Rishab F Tated, Sunku Rohan, Triveni S Pujar "CNN based Leaf Disease Identification and Remedy Recommendation System,” IEEE Xplore, ICEC 